Quim. Nova, Vol. 37, No. 1, 22-26, 2014

\title{
MÉTODO HIDROMETALÚRGICO PARA RECICLAGEM DE METAIS TERRAS RARAS, COBALTO, NÍQUEL, FERRO E MANGANÊS DE ELETRODOS NEGATIVOS DE BATERIAS EXAURIDAS DE Ni-MH DE TELEFONE CELULAR
}

\author{
Vinicius Emmanuel de Oliveira dos Santos*,a, Vinicius Guilherme Celante ${ }^{\mathrm{b}}$, Maria de Fátima Fontes Lelis ${ }^{\mathrm{a}}$ e Marcos \\ Benedito José Geraldo de Freitas ${ }^{\text {a }}$
}

${ }^{a}$ Departamento de Química, CCE, Universidade Federal do Espírito Santo, Av. Fernando Ferrari, 514, Vitória - ES, Brasil

'Instituto Federal do Espírito Santo - Campus Aracruz, Av. Morobá, 284, Morobá, Aracruz - ES, Brasil

Recebido em 20/02/2013; aceito em 30/08/2013; publicado na web em 09/10/2013

\begin{abstract}
HYDROMETALLURGICAL METHOD FOR RECYCLING RARE EARTH METALS, COBALT, NICKEL, IRON, AND MANGANESE FROM NEGATIVE ELECTRODES OF SPENT Ni-MH MOBILE PHONE BATTERIES. A hydrometallurgical method for the recovery of rare earth metals, cobalt, nickel, iron, and manganese from the negative electrodes of spent Ni-MH mobile phone batteries was developed. The rare earth compounds were obtained by chemical precipitation at $\mathrm{pH} 1.5$, with sodium cerium sulfate $\left(\mathrm{NaCe}\left(\mathrm{SO}_{4}\right)_{2} \cdot \mathrm{H}_{2} \mathrm{O}\right)$ and lanthanum sulfate $\left(\mathrm{La}_{2}\left(\mathrm{SO}_{4}\right)_{3} \cdot \mathrm{H}_{2} \mathrm{O}\right)$ as the major recovered components. Iron was recovered as $\mathrm{Fe}(\mathrm{OH})_{3}$ and $\mathrm{FeO}$. Manganese was obtained as $\mathrm{Mn}_{3} \mathrm{O}_{4}$. The recovered $\mathrm{Ni}(\mathrm{OH})_{2}$ and $\mathrm{Co}(\mathrm{OH})_{2}$ were subsequently used to synthesize $\mathrm{LiCoO}_{2}, \mathrm{LiNiO}_{2}$ and $\mathrm{CoO}$, for use as cathodes in ion-Li batteries. The anodes and recycled materials were characterized by analytical techniques.
\end{abstract}

Keywords: rare earth metals; batteries; recycling.

\section{INTRODUÇÃO}

As baterias Ni-MH têm sido ativamente pesquisadas desde 1950, como resultado do desenvolvimento de ligas de armazenamento de hidrogênio, mas não tiveram uso comercial até 1980. A preocupação ambiental cresceu no final da década de 1990, devido às restrições sobre a utilização de metais pesados, tais como chumbo, mercúrio, e cádmio. Isto levou ao desenvolvimento das chamadas baterias "verdes". De acordo com Zhang e colaboradores as baterias de Ni-MH foram desenvolvidas em 1989 e comercializadas principalmente no Japão em 1990. ${ }^{1}$ Estas baterias são ecologicamente corretas e podem substituir as baterias de Ni-Cd em muitas aplicações. As baterias secundárias de níquel-hidreto metálico são comumente usadas como fontes de energia em dispositivos eletrônicos, tais como: celulares, computadores e veículos elétricos híbridos. ${ }^{2,3}$ Há outras vantagens para as baterias Ni-MH, incluindo uma maior densidade de energia, taxas de carga/descarga mais elevadas, maior capacidade a baixa temperatura e a ausência de efeito memória. ${ }^{4,5}$ As baterias de íon - lítio foram produzidas pela Sony em 1991. Estas baterias têm substituído as baterias Ni-Cd e Ni-MH em muitas aplicações devido à sua alta densidade de energia, baixa taxa de auto-descarga e excelente ciclo de vida. Essas baterias também são aceitáveis em termos ambientais. ${ }^{6}$ A necessidade de baterias com alta eficiência energética que podem ser usadas com altas cargas e altas taxas de descarga tem aumentado o uso de baterias de íon - lítio. Como consequência, o consumo de baterias $\mathrm{Ni}-\mathrm{MH}$ para telefones celulares e notebooks diminuiu. $\mathrm{O}$ descarte de baterias exauridas de $\mathrm{Ni}-\mathrm{MH}$ tornou-se assim um assunto de interesse entre os pesquisadores.

O elétrodo positivo de $\mathrm{Ni}-\mathrm{MH}$ consiste em $\mathrm{NiOOH} / \mathrm{Ni}(\mathrm{OH})_{2}$, juntamente com aditivos, tais como: o grafite e o cobalto que melhoram o seu desempenho. Cobalto tem a finalidade de recobrir as partículas de hidróxido de níquel, aumentando a diferença de potencial entre a reação desejada de oxidação do hidróxido de níquel e a reação indesejável de desprendimento de oxigênio no eletrodo positivo. ${ }^{7}$ O eletrólito é geralmente hidróxido de potássio, e os separadores

*e-mail: viniciusemmanuel@hotmail.com consistem de poliamida, poliolefina ou uma mistura de ambos. ${ }^{8,9} \mathrm{O}$ elétrodo negativo das baterias de Ni-MH utiliza hidrogênio absorvido em ligas de tipo $A_{2}$ ou $A_{5}$. As ligas do tipo $A_{2}$ mais estudadas podem ser compostas por $\mathrm{Ti}-\mathrm{Zr}-\mathrm{V}-\mathrm{Ni}-\mathrm{Cr}$ e/ou outros elementos, onde $\mathrm{Ti}$ e $\mathrm{Zr}$ representam o componente A. ${ }^{10}$ Paul Ruetschi e colaboradores relatam uma composição definida para as ligas $\mathrm{AB}_{2}$ como: $\mathrm{V}_{15} \mathrm{Ti}_{15} \mathrm{Zr}_{20} \mathrm{Ni}_{28} \mathrm{Cr}_{5} \mathrm{Co}_{5} \mathrm{Fe}_{6} \mathrm{Mn}_{6}{ }^{11}$ Ligas $\mathrm{AB}_{2}$ possuem grande capacidade de armazenamento de hidrogênio e um melhor ciclo de vida do que as ligas convencionais do tipo $\mathrm{AB}_{5}$, contudo suas aplicações práticas são limitadas devido à energia de ativação lenta e elevado custo. ${ }^{12}$ Ligas $\mathrm{AB}_{5}$ contêm uma mistura de $\mathrm{La}, \mathrm{Pr}, \mathrm{Nd}, \mathrm{Ce}, \mathrm{Ni}$ e Co, conhecida comercialmente como mischmetal. ${ }^{13}$ As baterias de Ni-MH contêm quantidades significativas de metais valiosos, como os metais terras raras, Ni e Co. As quantidades de matérias-primas utilizadas na produção destas baterias são potencialmente muito grandes. ${ }^{14}$ Comercialmente as terras raras são usadas como aditivos em ligas de aço, ímãs permanentes, materiais de armazenamento de hidrogênio, discos de armazenamento ópticos, dispositivos magnéticos, raios catódicos eletroluminescentes e baterias domésticas. A importância de níquel no revestimento industrial é indicada pela grande quantidade de níquel que é consumida em cada ano em todo o mundo. Revestimentos de níquel são aplicados em automóveis, motocicletas, bicicletas e uma grande quantidade de bens de consumo. O cobalto é um metal crítico utilizado em várias aplicações industriais. $\mathrm{O}$ cobalto eletroquimicamente recuperado pode ser usado para fazer tanto ligas com propriedades magnéticas ou novos eletrodos para baterias. ${ }^{15}$ Assim, o seu preço tem aumentado consideravelmente devido à sua utilização como matéria-prima para os eletrodos positivos em baterias de íon - lítio. $\mathrm{O}$ alto consumo de cobalto levou à preocupação com o impacto ambiental devido à disposição inadequada de baterias no lixo municipal e tem levado muitos países a estabelecer normas sobre a prestação de tais produtos como as baterias. Como resultado, a reciclagem de baterias se tornou uma importante questão. ${ }^{16,17}$ Neste trabalho, um processo hidrometalúrgico para a reciclagem de metais de terras raras, níquel, cobalto, ferro e manganês presentes nos eletrodos negativos de baterias de telefones Ni-MH móveis foi desenvolvido. Os metais das terras raras foram recuperados como sais 
de sulfato. Níquel, cobalto, ferro e manganês foram recuperados como os seus hidróxidos ou óxidos. Posteriormente foi sintetizado a partir dos materiais recuperados, $\mathrm{Ni}(\mathrm{OH})_{2}$ e $\mathrm{Co}(\mathrm{OH})_{2}$, o composto $\mathrm{LiCoO}_{2}$, para confecção de cátodos de baterias de íon - lítio, sendo esta etapa a principal motivação deste trabalho. Os materiais recuperados foram caracterizados por difração de raios X (DRX), microscopia eletrônica de varredura (MEV), espectroscopia de infravermelho com transformada de Fourier (FT-IR) e com plasma indutivamente acoplado com espectroscopia de emissão óptica (ICP-OES).

\section{PARTE EXPERIMENTAL}

\section{Técnicas de caracterização}

Os espectros de difração de raios X (DRX) foram obtidos num difractômetro Shimadzu XRD-6000, modelo 20013. Foi usado radiação Ka de cobre com filtro de níquel correspondente a um comprimento de onda de $1.540 \mathrm{~nm}$ e velocidade de varredura de $0,5^{\circ}$ por minuto. As micrografias foram obtidas por microscopia eletrônica de varredura (MEV) em um modelo Shimadzu SSX-50. Na espectroscopia de emissão óptica com plasma indutivamente acoplado (ICP - OES), foi usado o equipamento Varian 715 - ES.

\section{Dissolução do elétrodo negativo e precipitação dos metais de terras raras}

As baterias exauridas de Ni-MH foram fisicamente desmontadas e separados em ânodos, cátodos, separadores orgânicos e compartimentos de aço. O ânodo foi dissolvido usando uma relação massa - volume de 1,0 g do material ativo por $20 \mathrm{~mL}$ em uma solução de $\mathrm{H}_{2} \mathrm{SO}_{4}$ 1,0 mol.L $\mathrm{L}^{-1}$ com 5,0 mL de $\mathrm{H}_{2} \mathrm{O}_{2} 30 \%$ v/v. A suspensão foi agitada constantemente a $298 \mathrm{~K}$ até a dissolução completa e, em seguida, filtrada. Os metais de terras raras, $\mathrm{La}$ e Ce, foram precipitados na solução por adição de $\mathrm{NaOH} 1,0 \mathrm{~mol} \mathrm{~L}^{-1}(\mathrm{pH} 1,5)$ a uma taxa de $0,10 \mathrm{~mL}$ por minuto com agitação constante. ${ }^{18}$ As alterações no $\mathrm{pH}$ e condutividade foram medidas durante a precipitação dos metais de terras raras. O precipitado foi lavado e seco à temperatura ambiente e armazenado em um dessecador.

\section{Recuperação de cobalto, níquel, ferro e manganês}

A mistura resultante foi filtrada após a precipitação dos compostos de terras raras. Em seguida, uma solução de $\mathrm{NH}_{4} \mathrm{OH}$ 6,0 mol L-1 e $\mathrm{HCl}$ 6,0 $\mathrm{mol} \mathrm{L}^{-1}$ foi adicionado à solução do filtrado para formar um tampão de pH 9,3. O ferro foi precipitado com hidróxido de sódio e separado por centrifugação a 2500 rpm durante 10 minutos. Na etapa seguinte, o precipitado de hidróxido de ferro foi lavado e seco. Uma solução de $\mathrm{Na}_{2} \mathrm{~S} 1.0 \mathrm{~mol} \mathrm{~L}^{-1}$ foi então adicionada ao filtrado para precipitar $\mathrm{CoS}, \mathrm{ZnS}$, NiS e MnS. Os compostos $\mathrm{MnS}$ e $\mathrm{ZnS}$ foram dissolvidos pela adição de uma solução $1,0 \mathrm{~mol} \mathrm{~L}^{-1}$ de $\mathrm{HCl}$. O CoS e NiS precipitados foram removidos por centrifugação a $2.500 \mathrm{rpm}$ durante 10 minutos. Na solução contendo os íons de $\mathrm{Zn}^{2+}$ e $\mathrm{Mn}^{2+}$ foi adicionado $\mathrm{NaOH}$ até $\mathrm{pH} 13$, precipitando o $\mathrm{Mn}^{2+}$ com a formação do complexo solúvel $\left[\mathrm{Zn}(\mathrm{OH})_{4}\right]^{2-}$.

Os precipitados $\mathrm{NiS}$ e $\mathrm{CoS}$ foram dissolvidos com $\mathrm{HNO}_{3}$ 15,0 mol L-1. Na solução contendo $\mathrm{Ni}^{2+} \mathrm{e} \mathrm{Co}^{2+}$ foi, em seguida, adicionado $\mathrm{NaOH}$ até atingir o pH 9,0 precipitando os hidróxidos de níquel e de cobalto. ${ }^{19}$

\section{Síntese do material para cátodo de baterias de íon - lítio}

O material do cátodo para as baterias de íon - lítio foi preparado usando um método de reação em estado sólido com o reagente analítico $\mathrm{LiCO}_{3}$ e os materiais recuperados $\mathrm{Ni}(\mathrm{OH})_{2}$ e $\mathrm{Co}(\mathrm{OH})_{2}$ numa razão de massa 2:1:1. Foi adicionada água à mistura de reagentes sólidos até obter uma pasta do material precursor formado. ${ }^{20} \mathrm{Em}$ seguida, o material precursor foi aquecido a $80^{\circ} \mathrm{C}$ durante 60 minutos e depois a $650{ }^{\circ} \mathrm{C}$ durante 120 minutos para produzir óxidos duplos de lítio cobalto e óxidos de lítio níquel. A Figura 1 representa o fluxograma do processo hidrometalúrgico dos materiais recuperados das baterias exauridas de Ni-MH.

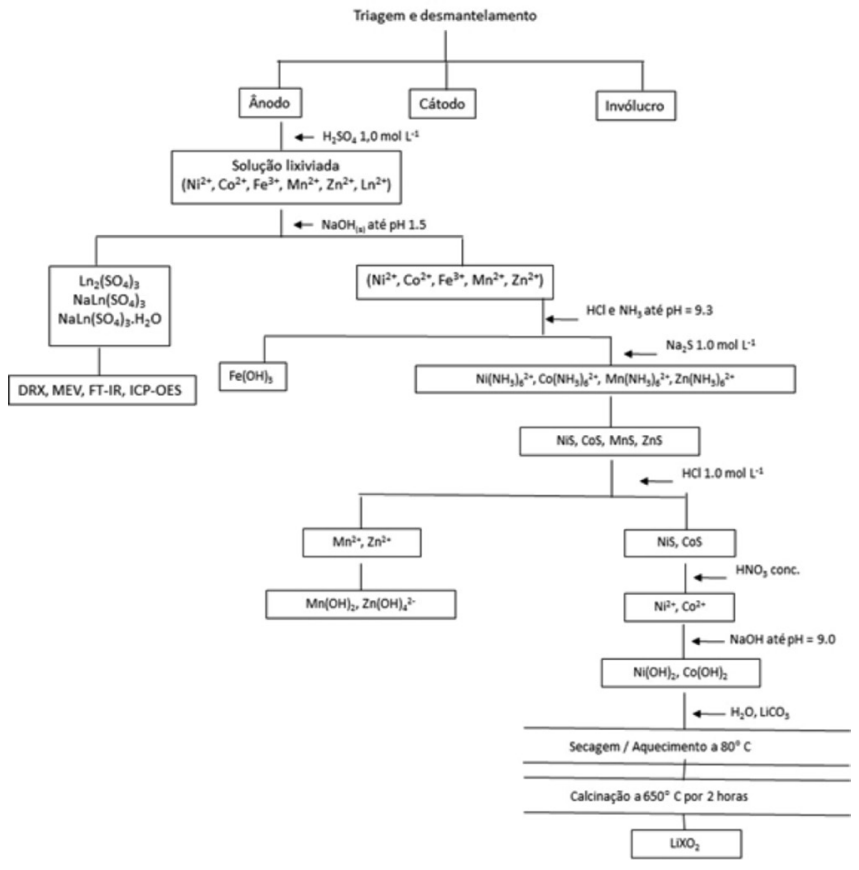

Figura 1. Fluxograma da rota de obtenção dos materiais recuperados da bateria de $\mathrm{Ni}-\mathrm{MH}$.(adaptado $)^{19}$

\section{RESULTADOS E DISCUSSÃO}

\section{Caracterização do ânodo da bateria de Ni-MH}

Os eletrodos negativos de baterias de Ni-MH recarregáveis foram caracterizados para determinar o melhor método para a recuperação das matérias-primas. As micrografias do elétrodo negativo são mostradas na Figura 2, nas quais podem ser observadas rachaduras ao longo de toda a superfície dos elétrodos negativos das baterias de Ni-MH.

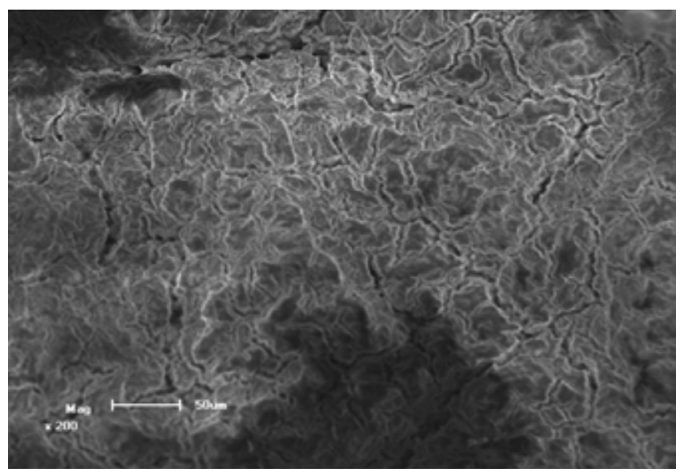

Figura 2. Micrografia do material do ânodo da bateria de Ni - MH. Aumento: $200 x$

As rachaduras ocorreram durante a remoção do material ativo devido ao processo de intercalação e desintercalação de átomos de 
hidrogênio (isto é, de carga e descarga, respectivamente, do ânodo). Na Figura 3 observa-se o difratograma de raios X do eletrodo negativo. Os sinais foram comparados com os cartões JCPDS identificando os compostos $\mathrm{CeO}_{2},{ }^{21} \mathrm{CeO},{ }^{22} \mathrm{Ce},{ }^{23} \mathrm{LaNi}_{5},{ }^{24} \mathrm{LaNi}^{25} \mathrm{FeNi},{ }^{26}$ $\mathrm{Mn}_{3} \mathrm{O}_{4}{ }^{27}$ e $\mathrm{CoONiO}{ }^{28}$

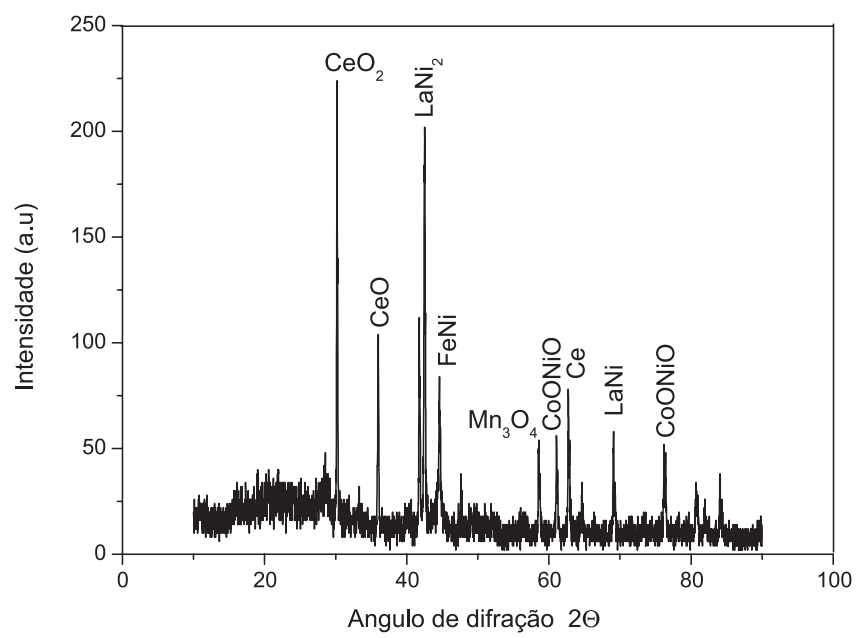

Figura 3. Difratograma de raios X do material do ânodo da bateria de $\mathrm{Ni}-\mathrm{MH}$

A concentração relativa do material do ânodo foi determinada por análise de ICP-OES e apresentou as seguintes concentrações: $\mathrm{Co}=302 \mathrm{ppm}, \mathrm{Ni}=190.0 \mathrm{ppm}, \mathrm{Ce}=138.8 \mathrm{ppm}, \mathrm{Fe}=84.1 \mathrm{ppm}, \mathrm{La}$ $=22.1 \mathrm{ppm}, \mathrm{Mn}=18.9 \mathrm{ppm}, \mathrm{Zn}=1.1 \mathrm{ppm}$. Os resultados indicam altas concentrações de $\mathrm{Co}$, Ni e Ce no qual é substituído pelo La em muitas ligas. $\mathrm{O} F e$ detectado é, devido à contaminação com o coletor de corrente, Mn e traços de Zn são provenientes do eletrodo positivo. Os resultados de ICP-OES concordaram muito bem com as medições de difração de raios $\mathrm{X}$.

\section{Caracterização dos compostos de terras raras recuperados}

A composição e a concentração relativa dos elementos químicos que compõem os materiais de terras raras foram $\mathrm{Ce}=24.67 \mathrm{ppm}$, $\mathrm{La}=6.256 \mathrm{ppm}, \mathrm{Mn}=0.089 \mathrm{ppm}, \mathrm{Zn}=$ não detectado, $\mathrm{Ni}=$ não detectado, $\mathrm{Co}=$ não detectado e $\mathrm{Fe}=$ não detectado. A análise de ICP-OES confirmou que os elementos cobalto, níquel e ferro permaneceram na solução após a precipitação de cério e lantânio e que existe uma baixa concentração de manganês no material recuperado. Os compostos de terras raras são recuperados com elevada pureza e podem ser usados em aplicações industriais. A Figura 4 mostra o difratograma de raios $\mathrm{X}$ para os materiais recuperados. De acordo com os arquivos JPCDS, os materiais recuperados são compostos por $\mathrm{NaCe}\left(\mathrm{SO}_{4}\right)_{2} \cdot \mathrm{H}_{2} \mathrm{O},{ }^{29} \mathrm{NaCe}\left(\mathrm{SO}_{4}\right)_{2},{ }^{30} \mathrm{Ce}_{2}\left(\mathrm{SO}_{4}\right)_{3},{ }^{31} \mathrm{NaCe}\left(\mathrm{SO}_{4}\right)_{2}{ }^{32} \mathrm{e}$ $\mathrm{La}_{2}\left(\mathrm{SO}_{4}\right)_{3} \cdot \mathrm{H}_{2} \mathrm{O} \cdot{ }^{33}$ Estes resultados estão de acordo com a solução de dissolução do ânodo analisado por ICP-OES.

$\mathrm{Na}$ Figura 5 pode-se ver a micrografia dos compostos de terras raras, sendo o tamanho de partícula inferior a 1,0 $\mu \mathrm{m}$ apresentando uma estrutura hexagonal clara. O espectro de infravermelho dos compostos de terras raras confirma que os compostos foram obtidos nas suas formas hidratadas com a presença de bandas na região de $3588 \mathrm{~cm}^{-1}$ e $1616 \mathrm{~cm}^{-1}$, relativas, respectivamente, à vibração de alongamento do grupo $\mathrm{OH}^{-}$e deformação da água. Além disso, a banda observada a $3510 \mathrm{~cm}^{-1}$ sugere a existência de água cristalizada, demonstrando a presença de uma forte banda entre $1108 \mathrm{~cm}^{-1} \mathrm{e} 1053 \mathrm{~cm}^{-1} \mathrm{e}$ uma banda fraca a $1003 \mathrm{~cm}^{-1}$, típicas de sulfato. As faixas compreendidas entre $675 \mathrm{~cm}^{-1}$ e $565 \mathrm{~cm}^{-1}$ indicam vibrações das ligações metal - oxigênio ( $\mathrm{La}-\mathrm{O}$ ou $\mathrm{Ce}-\mathrm{O})$.

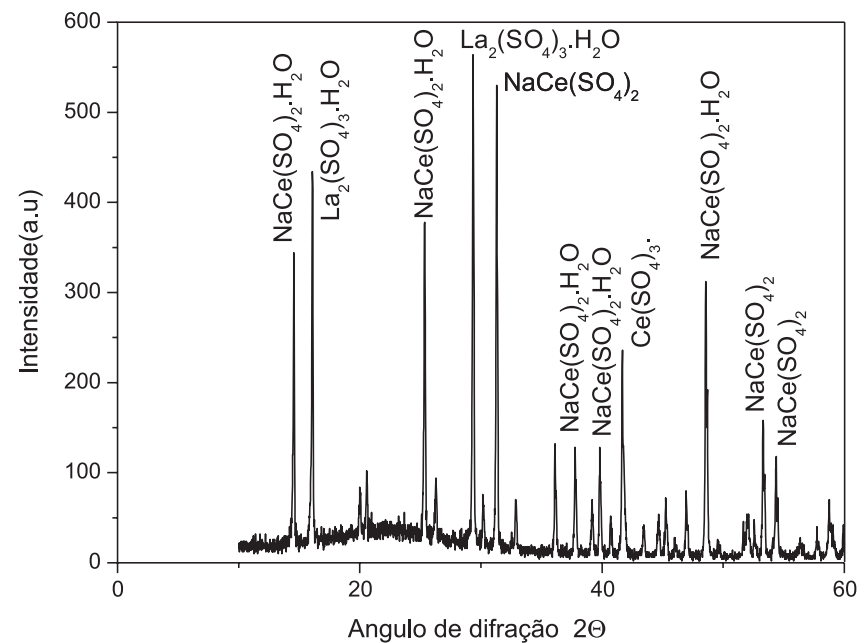

Figura 4. Difratograma de raios $X$ dos compostos recuperados das terras raras

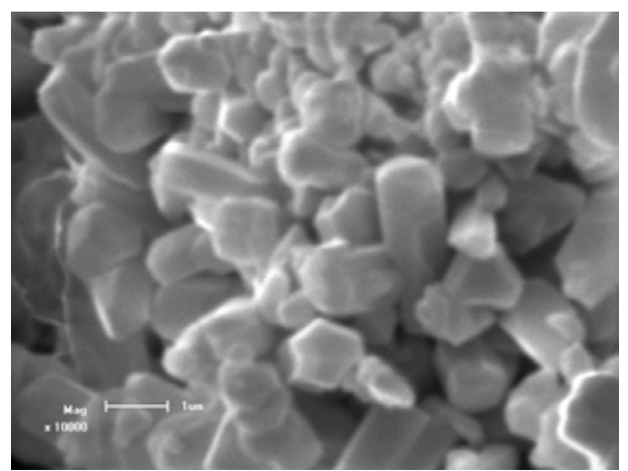

Figura 5. Micrografia dos compostos recuperados das terras raras. Aumento: $10000 x$

\section{Caracterização do cobalto e níquel recuperados}

O difratograma de raios - $\mathrm{X}$ dos compostos Co e Ni são mostrados na Figura 6. Este difratograma apresenta picos que, quando comparados com a base de dados JCPDS, indicam um empacotamento hexagonal de $\beta$ - $\mathrm{Ni}(\mathrm{OH})_{2}{ }^{34} \mathrm{e} \mathrm{Co}(\mathrm{OH})_{2},{ }^{35}$ com os parâmetros a $=3,13 \AA$ e c $=4,61 \AA$. A estrutura cúbica para o $\mathrm{CoO}^{36}$ apresenta parâmetro de rede $\mathrm{a}=4,29 \AA$.

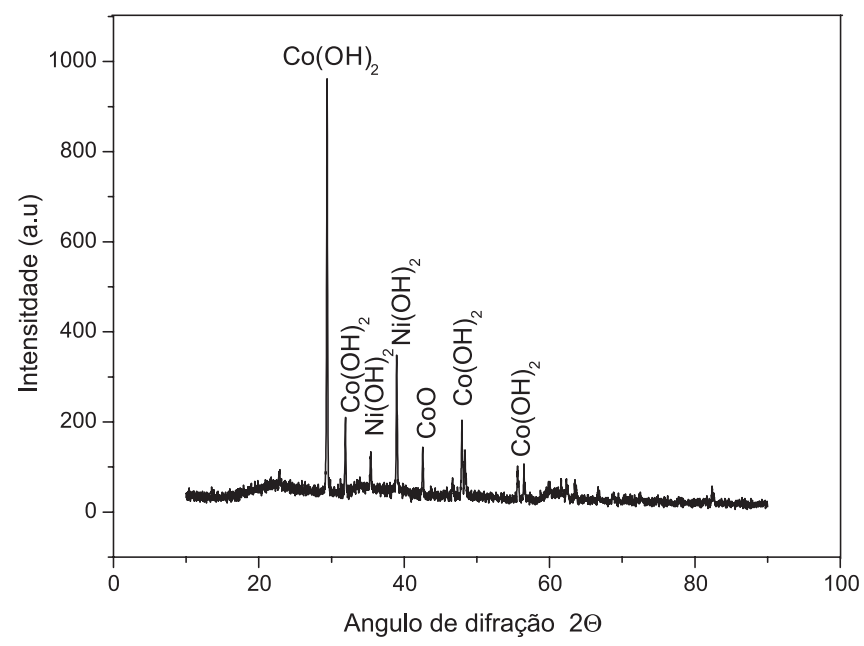

Figura 6. Difratograma de raios $X$ dos materiais recuperados dos metais níquel e cobalto 
No espectro de FT-IR, os números de onda de $3644 \mathrm{~cm}^{-1} \mathrm{e}$ $3464 \mathrm{~cm}^{-1}$ indicam a vibração dos grupos hidroxilas do $\mathrm{Ni}(\mathrm{OH})_{2} \mathrm{e}$ $\mathrm{Co}(\mathrm{OH})_{2}$ e o alongamento vibracional do grupo hidroxila da água adsorvida, respectivamente. Sinais de FT-IR de $1384 \mathrm{~cm}^{-1}$ são característicos de sulfatos e carbonatos. Estes resultados confirmam que o hidróxido de níquel e cobalto retém sólidos de sulfato e carbonato a partir das soluções iniciais. Além disso, a banda de absorção em $546 \mathrm{~cm}^{-1}$ é atribuível ao alongamento vibracional da ligação Co-O. O alongamento vibracional do Ni-O pode ser observado nas regiões de $637 \mathrm{~cm}^{-1}$ e $835 \mathrm{~cm}^{-1}$. Os hidróxidos de cobalto e níquel recuperados foram posteriormente utilizados para sintetizar materiais de cátodos para baterias de íon - lítio.

\section{Caracterização dos materiais sintetizados para baterias de íon - lítio}

Na Figura 7 observa-se o difratograma de raios $X$ dos materiais sintetizados. Os picos dos difratogramas foram comparados com os arquivos JPCDS para confirmar que os materiais recuperados são compostos de $\mathrm{LiCoO}_{2},{ }^{37} \mathrm{LiNiO}_{2}{ }^{38}$ e $\mathrm{CoO}$.

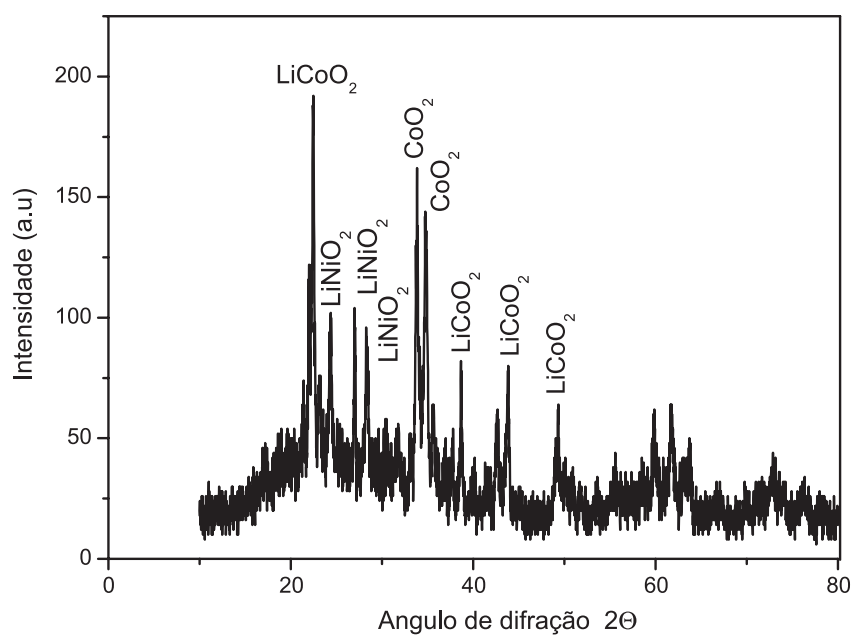

Figura 7. Difratograma de raios $X$ dos compostos sintetizados para cátodos de baterias de íon - lítio

O resultado de microscopia eletrônica de varredura detectou microporos e macroporos. Os microporos são necessários para melhorar a capacidade da bateria devido ao aumento da área de superfície na reação eletroquímica. Os macroporos asseguram a difusão dos íons de lítio no material do cátodo.

\section{Caracterização dos compostos de ferro recuperados por precipitação}

O difratograma de raios X (DRX) dos compostos de ferro recuperados por precipitação segundo os dados dos cartões JPCDS detectou que os picos nos difratogramas foram identificados como $\mathrm{Fe}(\mathrm{OH})_{3}{ }^{39}$ e $\mathrm{FeO}^{40}$ com empacotamento cúbico. A estrutura de $\mathrm{Fe}(\mathrm{OH})_{2}{ }^{41}$ existe como uma célula hexagonal, com parâmetros de rede $\mathrm{a}=3,27 \AA$ e $\mathrm{c}=4,62 \AA$. Além disso, uma estrutura de $\mathrm{Fe}_{2} \mathrm{O}_{3} \cdot \mathrm{H}_{2} \mathrm{O}^{42}$ é identificada em um sistema de empacotamento ortorrômbico com parâmetros de rede $\mathrm{a}=4,59 \AA, \mathrm{b}=9,94 \AA \mathrm{e} \mathrm{c}=3,015 \AA$.

\section{Caracterização dos compostos de manganês recuperados por precipitação}

O hidróxido de manganês precipitado foi submetido a um processo de envelhecimento na solução alcalina e na presença de oxigênio. Assim, por conseguinte, não é surpreendente que $\mathrm{Mn}_{3} \mathrm{O}_{4}$ foi obtido como resultado deste envelhecimento. Os materiais recuperados são constituídos por óxidos e óxidos hidróxidos de manganês. De acordo com os arquivos JPCDS o composto $\mathrm{Mn}_{3} \mathrm{O}_{4}$ possui um sistema de empacotamento tetragonal com parâmetros de rede: $\mathrm{a}=$ $5.76 \AA$ e c $=9.44 \AA$. A estrutura do composto $\mathrm{MnO}(\mathrm{OH})_{2}{ }^{43}$ possui um sistema de empacotamento hexagonal com parâmetros de rede: $\mathrm{a}=9.65 \AA$ e c $=4.43 \AA$. Os picos de difração são aumentados devido ao pequeno tamanho dos cristalitos analisados.

\section{CONCLUSÕES}

Os eletrodos negativos das baterias de Ni-MH recarregáveis foram caracterizados para determinar o melhor método para a recuperação dos materiais reciclados. De acordo com a análise de difração de raios $\mathrm{X}$, o eletrodo negativo apresentou as estruturas $\mathrm{CeO}, \mathrm{LaNi}_{5}, \mathrm{La}_{2} \mathrm{O}_{3}, \mathrm{Mn}_{3} \mathrm{O}_{4}, \mathrm{FeNi}, \mathrm{CoO}$ e NiO. Portanto, um eletrodo negativo do tipo $\mathrm{AB}_{5}$. As concentrações destes materiais anódicos foram determinadas por ICP-OES. A análise de ICP-OES revelou altas concentrações de $\mathrm{Co}$, Ni e Ce, que substitui o lantânio em muitas ligas. O ferro é devido à contaminação do coletor de corrente, $\mathrm{Mn}$ e $\mathrm{Zn}$ são elementos de traços do cátodo devido ao processo de prensagem. Um método hidrometalúrgico foi posteriormente desenvolvido para a recuperação de metais de terras raras, cobalto, níquel, ferro e manganês a partir de baterias de telefones celulares de Ni-MH em escala de laboratório. Os compostos de terras raras foram obtidos por precipitação química em pH 1,5 sendo o composto majoritário $\mathrm{NaCe}\left(\mathrm{SO}_{4}\right)_{2} \cdot \mathrm{H}_{2} \mathrm{O}$. A precipitação das terras raras é considerada quantitativa, uma vez que os metais La e Ce possuem baixa solubilidade em meio de íons $\mathrm{SO}_{4}^{2-}$ e o resultado de ICP-OES da solução sobrenadante não detectou estes metais. A composição e concentração relativa dos elementos químicos que constituíam os materiais de terras raras recuperados foram determinadas por ICP OES. Esta análise confirmou que o cobalto, níquel, ferro e manganês permanecem em solução após a precipitação do cério e lantânio. Os compostos de terras raras são recuperados com elevado grau de pureza e podem ser utilizados em futuras aplicações industriais. $\mathrm{O}$ ferro foi recuperado na forma de $\mathrm{Fe}(\mathrm{OH})_{3}, \mathrm{FeO}, \mathrm{Fe}_{2} \mathrm{O}_{3}, \mathrm{o} \mathrm{Fe}(\mathrm{OH})_{2}$ e $\mathrm{Fe}_{2} \mathrm{O}_{3} \cdot \mathrm{H}_{2} \mathrm{O}$. Manganês inicialmente passa por um processo de envelhecimento em solução alcalina e na presença de oxigênio produzindo o $\mathrm{Mn}_{3} \mathrm{O}_{4}$. Níquel e cobalto foram recuperados como $\beta-\mathrm{Ni}(\mathrm{OH})_{2}$ e $\mathrm{Co}(\mathrm{OH})_{2}$, com parâmetros de rede a $=3,13 \AA$ e c = $4,61 \AA$ A. Os hidróxidos de cobalto e níquel recuperados foram então usados para sintetizar os materiais $\mathrm{LiCoO}_{2}, \mathrm{LiNiO}_{2}$ e $\mathrm{CoO}$ utilizados em cátodos de baterias de íon - lítio.

\section{AGRADECIMENTOS}

À CAPES, CNPq e PETROBRAS, pelo apoio financeiro.

\section{REFERÊNCIAS}

1. Zhang, P.; Yokoyama, T.; Itabashi,O.; Wakui, Y.; Suzuki, T.M.; Inoque, K.; J. Power Sources 1999, 77, 116.

2. Rodrigues, L. E. O. C.; Mansur, M. B. J.; J. Power Sources 2010, 195, 3735.

3. Soria, M. L.; Chacón, J.; Hernández, J. C.; J. Power Sources 2001, 102, 97.

4. Bernades, A. M.; Espinosa, D. C. R.; Tenório, J. A. S.; J. Power Sources 2004, 130, 291.

5. Espinosa, D. C. R.; Bernardes, A. M.; Tenório, J. A. S.; J. Power Sources 2004, 135, 311. 
6. Kanamura, K.; Hoshikawa, W.; Umegaki, T.; J. Electrochem. Soc. 2002, $149,339$.

7. Afonso, J. C.; Valverde, M. I.; Barandas, B. G. M.; Quim. Nova. 2007, 30,712 .

8. Yinga,T. K.; Gaob, X. P.; Huc, W. K.; Wud, F.; Noréusc, D.; Int. J. Hydrogen Energy 2006, 31, 525.

9. Deabate, S.; Fourgeot, F.; Henn, F.; Electrochim. Acta 2006, 51, 5430.

10. Sullivan, J. L.; Gaines, L.; Energy Convers. Manage. 2012, 58, 134.

11. Ruetschi, P.; Meli, F.; Desilvestro, J.; J. Power Sources 1995, 57, 85.

12. Wen, M.; Chen, L.; Tong, M.; Chen, D.; Mater. Sci. Technol. 2001, 17, Suppl.1.

13. Lupi, C.; Pilone, D.; Waste Manage. 2002, 22, 871

14. Xiaofeng, LI.; Xia, B.; J. Alloys Compd. 2005, 391,190.

15. Freitas, M. B. J. G.; Pegoretti, V. C.; Pietre, M. K.; J. Power Sources 2007, 164, 947.

16. Zhang, P.; Yokoyama, T.; Itabashi, O.; Wakui, Y.; Suzuki, T. M.; J. Power Sources 1999, 77, 116.

17. Pietrelli, L.; Bellomo, B.; Montereali, D. M.; Hydrometallurgy 2002, 66, 135

18. Bertuol, D. A.; Bernardes, A. M.; Tenório, J. A.; J. Power Sources 2009, 193, 914.

19. Baccan, N.; Aleixo, L. M.; Stein, E.; Godinho, O. E. S; Introdução a Semimicroanalise Qualitativa, $4^{\text {th }}$ ed., Unicamp: São Paulo, 1990.

20. Jutang, S.; Wang, C.; Xiaoling, M. A.; Cheng, J.; Yunhong, Z.; Mater. Lett. 2007, 61, 556.

21. Joint Committee on Power Diffraction Standards (JCPDS), Card No. $34-0394$.

22. Joint Committee on Power Diffraction Standards (JCPDS), Card No. $33-0334$

23. Joint Committee on Power Diffraction Standards (JCPDS), Card No. $38-0763$

24. Joint Committee on Power Diffraction Standards (JCPDS), Card No. 12 - 0497.

25. Joint Committee on Power Diffraction Standards (JCPDS), Card No. $19-0654$

26. Joint Committee on Power Diffraction Standards (JCPDS), Card No. 03 -1044 .
27. Joint Committee on Power Diffraction Standards (JCPDS), Card No. 16 - 0154.

28. Joint Committee on Power Diffraction Standards (JCPDS), Card No. 03 - 0984.

29. Joint Committee on Power Diffraction Standards (JCPDS), Card No. $40-1485$.

30. Joint Committee on Power Diffraction Standards (JCPDS), Card No. $21-1109$.

31. Joint Committee on Power Diffraction Standards (JCPDS), Card No. 1- 208.

32. Joint Committee on Power Diffraction Standards (JCPDS), Card No. 21-1109.

33. Joint Committee on Power Diffraction Standards (JCPDS), Card No. 41- 509.

34. Joint Committee on Power Diffraction Standards (JCPDS), Card No. $14-117$.

35. Joint Committee on Power Diffraction Standards (JCPDS), Card No. 2 - 1094 .

36. Joint Committee on Power Diffraction Standards (JCPDS), Card No. $1-1227$.

37. Joint Committee on Power Diffraction Standards (JCPDS), Card No. 44 -145 .

38. Joint Committee on Power Diffraction Standards (JCPDS), Card No. $26-117$.

39. Joint Committee on Power Diffraction Standards (JCPDS), Card No. $22-346$.

40. Joint Committee on Power Diffraction Standards (JCPDS), Card No. 2 - 1186.

41. Joint Committee on Power Diffraction Standards (JCPDS), Card No. 3 - 903 .

42. Joint Committee on Power Diffraction Standards (JCPDS), Card No. $2-272$.

43. Joint Committee on Power Diffraction Standards (JCPDS), Card No. 17 - 510 . 